\title{
Animal Models to Study Emerging Technologies Against SARS-CoV-2
}

\author{
Jhinuk Basu Mullick, ${ }^{1}$ Chelsey S. Simmons $\left[0,{ }^{1,2}\right.$ and Janak Gaire ${ }^{1}$ \\ ${ }^{1}$ Department of Mechanical \& Aerospace Engineering, Herbert Wertheim College of Engineering, University of Florida, PO \\ Box 116250, Gainesville, FL 32611, USA; and ${ }^{2}$ Division of Cardiovascular Medicine, J. Crayton Pruitt Family Department of \\ Biomedical Engineering, University of Florida, Gainesville, USA
}

(Received 3 June 2020; accepted 16 July 2020; published online 27 July 2020)

Associate Editor Owen McCarty oversaw the review of this article.

\begin{abstract}
New technologies are being developed toward the novel coronavirus SARS-CoV-2 to understand its pathogenesis and transmission, to develop therapeutics and vaccines, and to formulate preventive strategies. Animal models are indispensable to understand these processes and develop and test emerging technologies; however, the mechanism of infection for SARS-CoV-2 requires certain similarities to humans that do not exist in common laboratory rodents. Here, we review important elements of viral infection, transmission, and clinical presentation reflected by various animal models readily available or being developed and studied for SARS-CoV-2 to help bioengineers evaluate appropriate preclinical models for their emerging technologies. Importantly, applications of traditional mice and rat models are limited for studying SARS-CoV-2 and development of COVID-19. Non-human primates, Syrian hamsters, ferrets, cats, and engineered chimeras mimic the human infection more closely and hold strong potential as animal models of SARS-CoV-2 infection and progression of resulting human disease.
\end{abstract}

Keywords-COVID-19, ACE2 receptor, Vaccine studies, Pathogenesis, Diagnostics, Transgenic models, Non-human primates, Domestic pets, Farm animals.

\section{ABBREVIATIONS}

ACE Angiotensin-converting enzyme

$\mathrm{CoV}$ Coronavirus

COVID-19 Coronavirus disease identified in 2019

Dpi Days post-inoculation

hACE2 Human ACE2

huPBMC Human peripheral blood mononuclear cells

K18 Human cytokeratin 18 promoter

mACE2 Mouse ACE2

Address correspondence to Chelsey S. Simmons, Department of Mechanical \& Aerospace Engineering, Herbert Wertheim College of Engineering, University of Florida, PO Box 116250, Gainesville, FL 32611, USA. Electronic mail: css@ufl.edu
MERS Middle east respiratory syndrome coronavirus identified in 2012

$\mathrm{NAb} \quad$ Neutralizing antibody

NSG NOD SCID gamma

SARS Severe acute respiratory syndrome coronavirus identified in 2003

TMPRSS Transmembrane protease, serine

\section{INTRODUCTION}

The novel coronavirus SARS-CoV-2, which emerged in December 2019 and causes the disease "coronavirus disease '19" (COVID-19), has galvanized biomedical research to understand, prevent, and treat this life-threatening condition. ${ }^{43}$ As with many diseases, animal models are being leveraged to study cellular pathogenesis and transmission of infection, as well as to examine the efficacy of viral vaccines and analyzing herd immunity post-vaccination. However, unique features of SARS-CoV-2 limit the utility of traditional laboratory animals.

SARS-CoV-2 has a solar-corona-like appearance with spike surface proteins, the characteristic namesake of coronaviruses. These spike proteins have two subunits: the surface unit S1 binds with high affinity to human angiotensin-converting enzyme 2 (ACE2) receptors and the transmembrane unit S2, which is then cleaved by human transmembrane serine proteases TMPRSS1 and TMPTSS2. ${ }^{24}$ ACE2 and TMPRSSs co-expression are essential for SARS-CoV2 infection, but mice, rabbits, rats, and guinea pigs do not have ACE2 receptors susceptible to SARS-CoV-2 binding. ${ }^{49}$ Furthermore, due to their anatomical difference and small size, intranasal viral inoculation of typical small rodent models can result in inhalation 
and ingestion, thus making it difficult to discriminate between intranasal, oral, and intrapulmonary vaccination $^{31}$ and limiting their utility as a model of vaccination against SARS-CoV-2. Here, we discuss alternative rodent models, large animals, and chimeras that may be useful to the bioengineering community working on innovative treatments and vaccinations against SARS-CoV-2.

\section{Important Factors for Selecting an Animal Model to Investigate SARS-CoV-2}

As with most diseases, no single animal model perfectly matches the clinical profile of SARS-CoV-2, as observed in humans. A necessary condition for animal models to model the cellular pathogenesis of SARS-CoV-2 infection is ACE2 receptor and serine proteases similar to humans, as discussed above. Several larger mammals such as palm civets, pigs, ferrets, cats, and nonhuman primates have ACE2 receptors which the virus can recognize. ${ }^{54}$ However, many animals known to be susceptible to similar coronaviruses, e.g., chickens, pigs, and dogs, ${ }^{19}$ have not been reported to be susceptible to SARS-CoV-2.

Given the nuances of each animal model, the choice of animal model for studying COVID-19 should vary based on the application. To investigate treatments, for example, models should be considered that show characteristic symptoms similar to humans such as shortness of breath, fever, weight loss, loss of appetite, and pneumonia. ${ }^{20}$ Since older humans have exhibited increased susceptibility to SARS-CoV- $2^{33}$ models utilizing aged adult animals should be favored. For genomic and pathological studies, viral RNA or histopathological tissues need to be obtained, for which animal models should be preferred in whom similar organs as those affected during human pathogenesis are involved. For COVID-19, these organs include the respiratory tract, lungs, and gastrointestinal tract. Mode of transmission must be similar for projects working to develop personal protective equipment such as respiratory masks or eye protection.

In such urgent times, large animal and non-traditional rodent models can be readily utilized without the time delay associated with developing transgenic and chimeric small animal models. Large mammals also have the advantage of physiological, anatomical and immunological proximity to humans. ${ }^{16}$ Their mucosal surfaces can be easily accessed for intranasal and oral vaccine administration and, often being outbred species, may yield more clinically relevant results. A brief overview of readily available animal models of COVID-19 is provided in Table 1.

\section{RODENT MODELS OF COVID-19}

\section{Golden Syrian Hamsters}

Hamsters are adept at amplifying many viruses and often studied for viral persistence and shedding. ${ }^{4}$ The golden Syrian hamster (Mesocricetus auratus) had been used previously to study other respiratory viruses such as Severe Acute Respiratory Syndrome coronavirus (SARS), adenovirus, and influenza virus. The structure of the ACE2 receptor of hamsters is similar to humans, allowing the spike protein of SARS-CoV-2 to bind with high affinity and generating preference for the Syrian hamster as a model in both SARS and SARSCoV-2 studies. To mimic human transmission, recent studies challenged male and female Syrian hamsters intranasally. ${ }^{10,45}$ The viral loads were found to be highest in the lungs at 2 days post-inoculation (dpi) and were reduced below the detection limits by $7 \mathrm{dpi}$, when the animals started to recover. The animals showed passive immunization whereby hamsters developed neutralizing antibodies (NAbs) by $7 \mathrm{dpi}^{10}$ or by $14 \mathrm{dpi}^{45}$ High transmission rate, as shown by transmission to all the naïve co-housed hamsters, did mimic the pathogenesis in humans and was likely through respiratory droplets or oral-fecal contamination. Despite high viral loads observed throughout many organs, the hamsters showed only mild symptoms such as weight loss, lethargy, ruffled fur, hunched back posture, and rapid breathing. ${ }^{10}$

The main disadvantage of the Syrian hamster model was the lack of mortality which did not match the human clinical profile. These studies also used younger hamsters between 4 and 10 weeks of age, likely following previous SARS-CoV experiments. ${ }^{39}$ However, in light of known complications in elderly patients, ${ }^{33}$ it may have resembled human disease more closely if aged hamsters were used. Overall, the studies show that the Syrian hamster is a useful animal model for SARS-CoV-2 infection especially to study viral replication, shedding, and transmission through the respiratory tract.

\section{Ferrets}

Having anatomical and physiological similarity with the human respiratory system, ferrets (Mustula putorius furo) have previously been used for studying SARS infection. ${ }^{56}$ Recent reports suggest affinity of SARS-CoV-2 for binding to ferret ACE2, and the mechanism of transmission correlated with human transmission, which required direct physical contact with potential airborne transmission as viral RNA was 


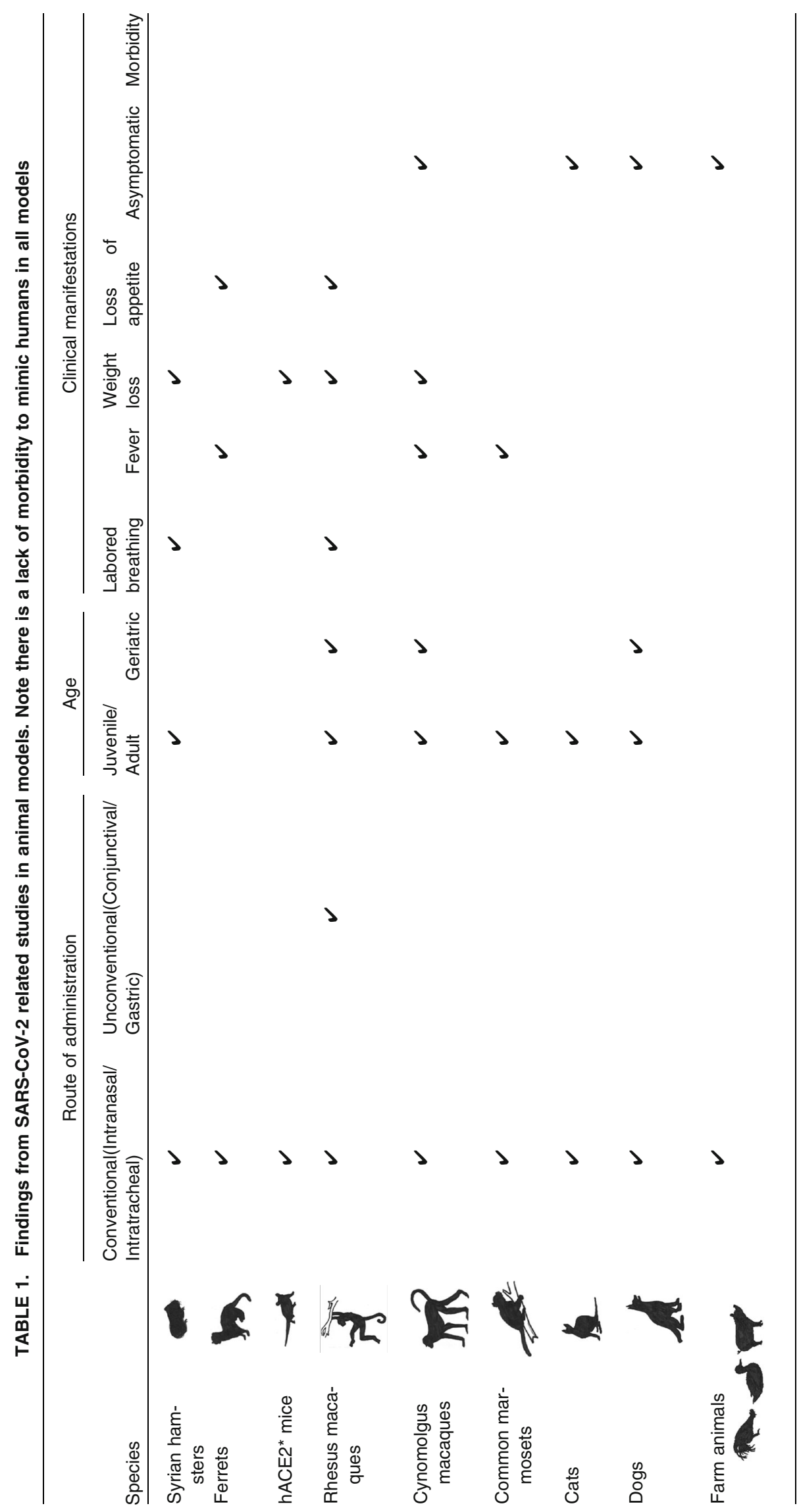


Mullick et al.

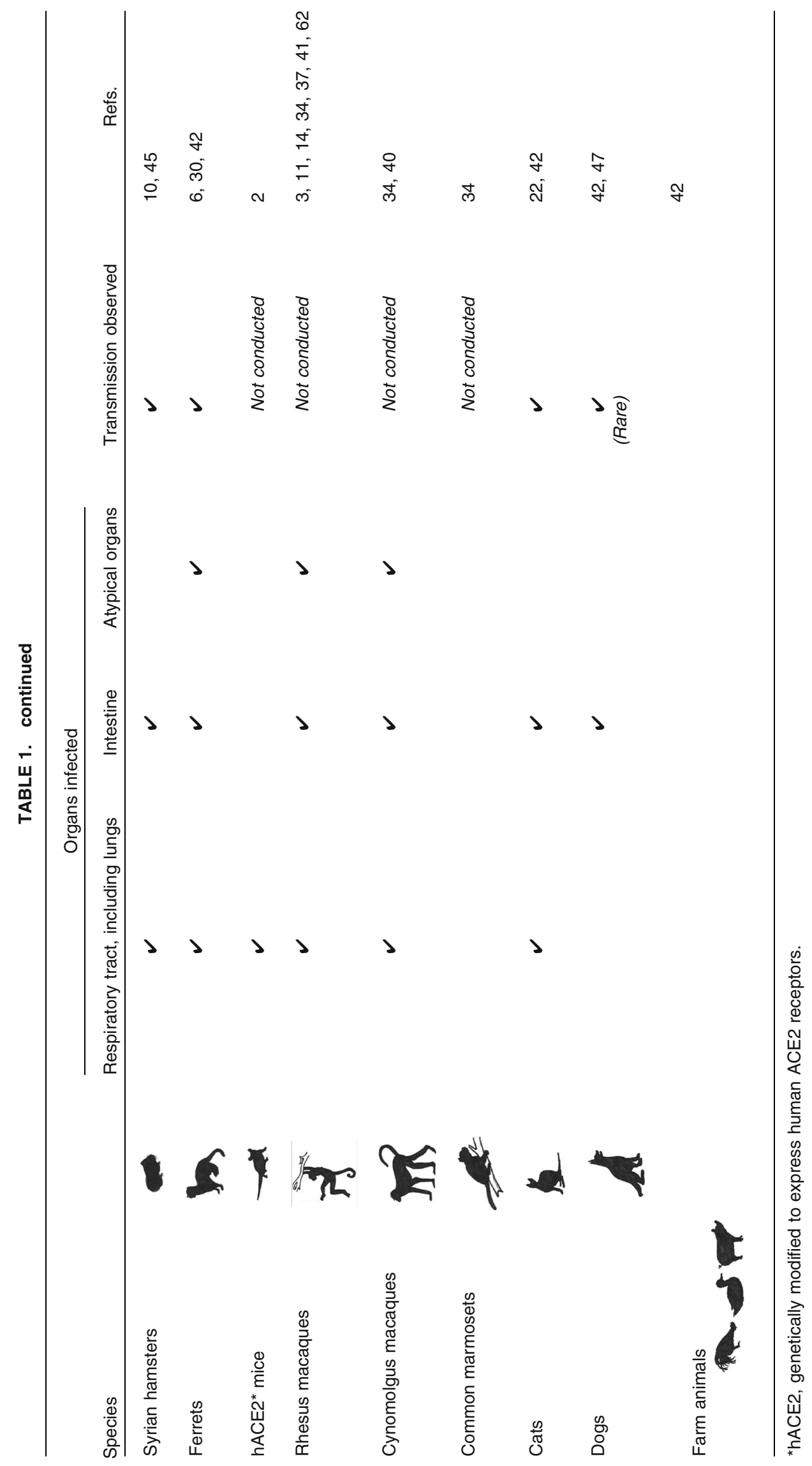


detected in nasal washes, saliva, blood, fecal, and urine samples. ${ }^{30,42}$ Both in humans and ferrets, the virus affected similar organs such as the nasal turbinate, trachea, lung, soft palate, tonsils, intestine, and kidney. ${ }^{6,30,42}$ Clinical signs were mild compared to human patients and included fever and loss of appetite. NAbs were detected starting $13 \mathrm{dpi}^{42}$ Blanco-Melo et al. analyzed the transcriptional regulation of immune modulators to SARS-CoV-2 in ferret model and inferred that severity of COVID-19 in aged population was linked with a severe inflammatory response. ${ }^{6}$ They suggest that controlling the SARS-CoV-2-associated cytokine storm $^{59}$ should be the primary focus of treatment.

The ferret thus can serve as a useful model for studying the pathogenesis of SARS-CoV-2 infection in the respiratory tract. Ferrets have been considered to be outbred species and hence also used widely in vaccination studies. However recent findings suggest that due to inbreeding, ferrets can be clustered based on geographical locations, where North American and Australian clusters have been found to be very low in genetic diversity. ${ }^{21}$ Researchers using ferrets as a model should consider these issues of the genetic background when interpreting their findings.

\section{Mice}

SARS and SARS-CoV-2 viruses cannot bind to mouse ACE2 (mACE2) due to differences in key amino acid residues. ${ }^{49}$ Due to this low sensitivity of mice to SARS viruses, several transgenic mice lines were created during the SARS epidemic to study its pathogenesis. Human ACE2 (hACE2) genes were expressed under a hybrid chicken beta-actin promoter with cytomegalovirus enhancer ${ }^{51}$ or cytokeratin 18 (K18) promoter. ${ }^{35}$ The Jackson Laboratory (Bar Harbor, $\mathrm{ME}$ ) has recently started re-producing the K18-hACE2 strain used to study SARS infection, ${ }^{55}$ although systemic damage and neuroinflammation still did not represent the human clinical profile. ${ }^{49}$ Yang et al. developed an efficient model where the endogenous mouse ACE2 promoter was used and had tissue distribution similar to the natural hACE2 distribution; however, this model still could not mimic the high mortality observed in humans. ${ }^{58}$

Since both the viruses have a similar mechanism of entry through the ACE2 receptor, the developed models are still relevant for SARS-CoV-2 study. Bao et al. used the mACE2 promoter line to study the pathogenesis of SARS-CoV-2 infection. ${ }^{2}$ Viral RNA was detected in the lungs, bronchi, and alveoli, while the wild-type mice failed to be infected by SARS-CoV2. The study thus found that the ACE2 receptor was essential for SARS-CoV-2 infection, as expected.
Soldatov et al. have been working on producing a transgenic line where hACE2 and hTMPRSS2 are coexpressed with the mice TMPRSS2 promoter through gene editing using CRISPR/Cas9. ${ }^{49}$ The group hypothesizes that the co-expression will make the model mimic human pathogenesis more closely, particularly in the lungs.

Other transgenic mice models studied during SARS infection can also be helpful for SARS-CoV-2 studies. One such model is the Ace2 knockout mouse, which lacks the ACE2 receptor and upon SARS infection has been seen to suffer from acute lung failure. ${ }^{27}$ TMPRSS2 knockout mice have shown lower levels of inflammatory cytokines and infiltration by immune cells, leading to less severe tissue damage in the lungs after SARS and Middle East Respiratory Syndrome coronavirus (MERS) infections, ${ }^{28}$ which has pointed researchers to TMPRSS2 as a potential therapeutic target for serine protease inhibitors. Additional strains such as STAT1 knockout, BALB/c, C57BL/6, and 129S6 mice that were studied for SARS may prove to be useful as well. ${ }^{50}$ The immune system of feral mice or "dirty mice", mice exposed to a diverse group of microbes, have broad $\mathrm{T}$ cell distribution that could rapidly control pathogens ${ }^{18}$ and render them potential candidates to gain insights into vaccine development for SARS-CoV-2.

\section{NON-HUMAN PRIMATE ANIMAL MODELS}

As close evolutionary relatives to humans, non-human primates have very similar physiology and immunology to humans ${ }^{31}$ and hence greater translational potential. Issues with non-human primate models, however, include additional ethical justifications, scarcity of biosafety level-3 facilities, and lack of trained personnel to carry out work. ${ }^{10}$ Nonetheless, many primate models have yielded helpful insights into SARS-CoV-2.

\section{Rhesus Macaques}

The rhesus macaque (Macaca mulatta) is broadly popular for studying re-infection potentials and designing vaccines. When infected with SARS-CoV-2, specifically, the rhesus macaque model manifests mild to moderate symptoms of decreased appetite, weight loss, fever, and increased or irregular respiration patterns along with hunched posture. ${ }^{3,11,34,37}$ Viral RNA can be detected throughout the respiratory and gastrointestinal tract, ${ }^{3,11,34,37,41}$ mimicking humans, and also from atypical organs such as spinal cord, heart, skeletal muscles, and bladder ${ }^{3}$; liver and kidney ${ }^{11,14}$; and the spleen. ${ }^{34}$ The hallmarks of human SARS- 
CoV-2 infection of pulmonary edema and diffused interstitial pneumonia were observed on radiographs throughout these studies. In age-related comparative studies, $\mathrm{Yu}$ et al. studied the effects of SARS-CoV-2 infection with three "young" (3-5 years) and two "geriatric" (15 years) animals. ${ }^{62}$ They found geriatric macaques suffered from more severe interstitial pneumonia with more viral load in lungs and anus. The older macaques had viral replication in the entire lung while the younger ones had only in the upper lobes of the lung. Another age-related study found that older animals had higher levels of virus-specific antibodies which were detectable early, by $4 \mathrm{dpi}^{34}$ Rhesus macaques were also used to investigate uncommon transmission routes; Deng et al. inoculated SARSCoV-2 through conjunctival and gastric routes. ${ }^{14}$ Though SARS-CoV-2 failed to replicate via the gastric route, mild interstitial pneumonia and alimentary canal infection were observed in the conjunctival route challenge.

Rhesus macaques also have been used to observe if primary infection with SARS-CoV-2 can protect against re-infections. In these studies, viral loads measured by bronchoalveolar lavage, nasopharyngeal swabs, or anal swabs were found to be reduced manyfold upon reinfection. ${ }^{3,11}$ Chandrashekar et al., upon rechallenging the animals, found viral loads were reduced $>100,000$-fold. ${ }^{11}$ Similar results were observed by Bao et al., where no clinical symptoms were observed upon reinfection. ${ }^{3}$ In a vaccine screening study, $\mathrm{Yu}$ et al. developed DNA vaccine candidates against SARS-CoV-2 spike protein, which were tested on 35 adult rhesus macaques. ${ }^{61}$ After vaccination and upon a second exposure, viral loads were reduced $>1,000$-fold. In all studies, animals developed NAbs.

Overall, the rhesus macaque model has been similar in many aspects to the human COVID-19 pathogenesis. Nonetheless, though studies utilized adult animals up to 15 years old, they could not mimic the high fatality rate of humans. $3,11,37$

\section{Cynomolgus Macaques}

The crab-eating macaque (Macaca fascicularis), originally found in Southeast Asia, had been studied previously for SARS. ${ }^{17}$ Similar to Rhesus macaques, when infected with SARS-CoV-2, geriatric animals showed prolonged viral shedding from the upper respiratory tract in comparison to younger animals through $21 \mathrm{dpi}^{40}$ However, animals infected with SARS-CoV-2 showed no obvious clinical signs or mortality, even in the geriatric group, ${ }^{40}$ which is in contrast to severe systemic responses seen in older macaques for SARS. ${ }^{48}$ When comparing male and female macaques, no difference in levels of antibodies was observed, though NAbs could be detected as early as $4 \mathrm{dpi}^{34} \mathrm{Lu}$ et al. did report fever and weight loss, and both studies reported diffuse interstitial pneumonia, a common complication in humans. ${ }^{34,40}$

Early and prolonged virus shedding of SARS-CoV2 compounded with the lack of symptoms highlights an area of concern for community transmission among humans as well. While macaques do not mimic the severity of human symptoms, they may still be helpful to understand disease progression in older and potentially asymptomatic subjects and thus to formulate strategies for containment of COVID-19.

\section{Common Marmosets}

Common marmosets (Callithrix jacchus), a new world monkey, were widely used to understand the pathogenesis of and immunization against the deadly MERS as their pathophysiology mimicked lethal pneumonia seen in humans. ${ }^{9}$ Its utility as a model to study SARS-CoV-2 has not been explored much, though. A single comparative study by $\mathrm{Lu}$ et al. was found in which six marmosets were intranasally infected with the virus for comparison with other nonhuman primates. ${ }^{34}$ Viral RNA was observed till $14 \mathrm{dpi}$ in blood and nasal, throat, and anal swabs; however, viral-specific antibodies could not be detected in serum, which could be due to lack of marmoset specific antibody detection kit. Only one-third of the marmosets had elevated body temperature, lung tissue showed no indication of pneumonia, and no other organs showed the presence of viral RNA. Overall, the common marmosets were found to be relatively resistant to SARS-CoV-2 as compared to rhesus macaques and cynomolgus macaques.

\section{DOMESTIC ANIMALS}

\section{Cats}

There has been evidence of infection with SARSCoV-2 in tigers at the Bronx Zoo and isolated reports of transmission from humans to domestic cats. ${ }^{25}$ It is helpful therefore, to look into the infectivity of SARSCoV-2 on cats to prevent chain of transmission between cats and humans. Cats previously had been found susceptible to SARS infection and to have the ACE2 receptor important in the development of COVID-like symptoms. ${ }^{52}$ More recently, Shi et al. inoculated seven 6-9 months-old "sub-adult" cats intranasally with a SARS-CoV-2 viral strain. ${ }^{42}$ Viral RNA was detected in the nasal turbinates, soft palates, tonsils, tracheas, and the small intestine on 3-6 dpi. The group also found transmission through droplets to 
the exposed cats. In 70-100 days-old "juvenile" cats, by $3 \mathrm{dpi}$, massive viral lesions were found in the nasal cavity, trachea and lungs, indicating that juvenile cats allow for better replication of the SARS-CoV-2 virus. NAbs were detected in the sub-adult cats between 11 and 12 dpi and in the juvenile cats between 10 and 20 dpi. In a similar study by Halfmann et al., inoculated cats showed viral shedding in nasal swab by $1-3 \mathrm{dpi}^{22}$ Transmission was also observed in cats that were cohoused. All tested cats were asymptomatic with no clinical symptoms of fever or weight loss. All inoculated and transmission-infected cats produced antiSARS-CoV-2 IgG antibodies at $24 \mathrm{dpi}^{22}$ In another study, it was reported that $14.7 \%$ of cats who were exposed to infected humans harbored antibodies against SARS-CoV-2 spike protein. ${ }^{63}$ The above studies indicate that cats are susceptible to the virus and may harbor it without showing any evident clinical symptoms or mortality. Since humans are in close contact with domesticated cats, there is therefore a risk of transmission to humans from asymptomatic feline companion animals.

\section{$\operatorname{Dog} S$}

Canine ACE2 is similar to hACE2, ${ }^{19}$ and though dogs are known to be infected by some coronaviruses, SARS-CoV-2 does not seem to be a concern in canines. The first suspected case of human-to-animal transmission of SARS-CoV-2 was in a Pomeranian dog belonging to an infected person in Hong Kong. ${ }^{47}$ Generally, isolated cases of SARS-CoV-2 infection in companion canines have found dogs to remain asymptomatic though viral RNA has been found in nasal, oral and rectal swabs. ${ }^{47}$ Shi et al. inoculated five 3-month-old beagles with SARS-CoV-2 intranasally, and two un-inoculated beagles were co-housed for transmission studies. ${ }^{42}$ While viral RNA was observed in rectal swabs of three virus-inoculated dogs by 2-6 dpi, viral RNA was not observed in any other organ. By the $14 \mathrm{dpi}$, two virus-inoculated dogs produced antibodies while the other dogs were found to be seronegative. Overall, results from these studies and broad testing of pets ${ }^{26}$ indicate low susceptibility for SARS-CoV-2 among dogs, suggesting a minimal risk of asymptomatic transmission from canine companion animals to humans.

\section{Farm Animals}

The first coronavirus infections were identified in chickens in the 1930s, thirty years before the discovery of the disease in humans. ${ }^{8}$ However, the current pandemic-causing coronavirus SARS-CoV-2 has been reported to be non-infective towards chickens, ducks, and pigs. ${ }^{42}$ In both inoculated animals and co-housed uninoculated animals, no viral RNA could be isolated from swabs. All animals were also found to be seronegative by $14 \mathrm{dpi}$. In horses, the enteric equine coronavirus has surged over the last few years, but there is no evidence horses are susceptible to infection with SARS-CoV-2. ${ }^{13}$ While farm animals are unlikely to transmit SARS-CoV-2, extensive outbreaks have been documented at meat processing plants because of the close working conditions among human workers, suggesting that food production will likely remain a concern throughout the pandemic. ${ }^{15}$

\section{HUMANIZED MICE AND OTHER CHIMERAS}

\section{Humanized Mice}

Despite highly conserved genes broadly, therapeutic and immunization strategies involving adaptive immunity are hard to translate from traditional animal models like mice, rats, and non-human primates into humans. To address these limitations, various strains of immunodeficient mice have been leveraged to create chimeras by transplanting cells and tissues of interest from humans. ${ }^{12,38,44}$ In particular, engineered chimeras incorporating human immune cells tend to better recapitulate the human immune system, enabling human-specific immune responses to deadly viruses. For example, humanized mice created by injecting human peripheral mononuclear cells (PBMCs) or human CD34+ cells into NOD scid gamma (NSG) mice, commonly known as huPBMCNSG or CD34+ humanized mice, respectively, have been a valuable tool for studying viral infections including HIV, ${ }^{29}$ Dengue, ${ }^{36}$ and Hepatitis. ${ }^{5}$ As an example, Kim et al. created huPBMC-NSG mice and infected with HIV-1 to test the effectiveness of antiviral drugs and the usefulness of NAbs. ${ }^{29}$ Following infection, the authors observed an increase in plasma viral load and a decrease in CD4+ T cells. After the use of an antiviral drug and $\mathrm{a}$ NAb in HIV-infected huPBMC-NSG mice, they observed a decrease in plasma viral loads and no decline in CD4+ T cells, demonstrating the effectiveness of intervention strategy.

Although we have not identified reports using humanized mice to study COVID-19, success from the aforementioned viral studies highlight the relevance and utility of humanized mice for COVID-19 research. CD34+ humanized mice and PBMC humanized mice are available from commercial vendors such as the Jackson Laboratory, and humanized mice can be further tailored according to research applications. Recently, Wahl et al. created mice with human lung tissue 
by subcutaneous transplantation of human fetal lung in NSG mice and later combined these mice with bone marrow-thymic-liver mice, a mouse line with a very robust human immune system. ${ }^{53}$ Thus, they created a humanized mouse containing both the human immune system and human "lungs" that could potentially be used for studying pathogenesis of respiratory viruses like SARS-CoV-2 and screening for antiviral drugs and therapeutic vaccines.

Humanized mice, though being the closest replication of the human immune system in a small animal model, come with some limitations. While mice with a degree of "humanized" immune function are available commercially, it will take additional effort to develop enhanced variants of humanized mice with a more functional human immune system, e.g. with human leukocyte antigens. ${ }^{7}$ Unlike readily available small animal models with a competent immune system, it takes a longer time to develop humanized mice and requires additional aseptic handling practices and pathogen-free facilities. Nonetheless, humanized mice offer a promising test-bed for rapid development and testing of antiviral drugs, vaccines, and their delivery related to COVID 19 research.

\section{Bat Mice}

Inspired by the success of using humanized mice to study species-specific response in small animal models, researchers have leveraged the utility of immunodeficient mice to develop chimeras using immune cells from complex animals such as bats. Bats are thought to harbor a number of viruses dangerous to humans and other animals without effect, but how viruses and the bat immune system coexist asymptomatically is largely unknown. Moreover, SARS-CoV-2 has $\sim 90 \%$ similarities to beta-coronaviruses isolated from bats, suggesting bat-to-human transmission was at the nexus of the current pandemic. In vivo studies of bats are limited, though, by challenging breeding conditions and long gestation periods ${ }^{23}$ concerns with capturing large numbers of conserved wild bats, ${ }^{46}$ and other challenges of outbred species. Most studies on bats are limited to specialized bat cell lines, ${ }^{1,64}$ and limits to the availability of these bat-specific cell lines and batspecific antibodies make even in vitro research more challenging than anticipated. To mitigate some of these challenges, a chimera model has been developed by Yong et al. that stably expresses the immune system of the bat (Eonycteris spelaea) on a mouse background. ${ }^{60}$ These "bat-mice" have been developed by transplanting bat bone marrow cells into immunodeficient NSG mice. This study showed that $\sim 80$ to 100 bat-mice can be generated from one bat, thus reducing problems associated with animal numbers. The bat-mice model reconstituted all major immune cells including monocytes, T and B lymphocytes, Natural Killer cells, and dendritic cells. The model has been found to generate bat specific antibodies in response to antigens as well as resistance to graft rejection when transplanted with bat cells even after 40 weeks.

The development of bat-mice has opened up new avenues for research that were previously deemed challenging due to the limited availability of bats for immunological research. A better understanding of how the bat's immune system handles various pathogens, including deadly viruses such as SARS-Cov-2, will provide valuable insights to strategize the development of effective therapeutic and preventative approaches and additional understanding of bat-tohuman transmission of a variety of viruses.

\section{FUTURE DIRECTIONS}

An efficient COVID-19 animal model which closely resembles the human clinical picture can accelerate the path for vaccine development and therapeutics as well as shine light on viral pathogenesis and formulation of preventive strategies. For most diseases, mice and other small rodents have been modified to cater to various demands of the research community. An abundant range of reagents and assays are available, too, for such analysis. The problem specific to SARSCoV-2, however, has been the dominant role of the ACE2 receptor in COVID-19 pathogenesis. The virus so far has not been found to bind to the ACE2 receptor of any of the commonly used small animals unless genetically modified. Genetic modification is a timeconsuming process, and the rapid spread of SARSCoV-2 infection and its compounding socioeconomic effects require more rapid solutions. Thus, researchers are shifting interest toward readily available larger animal models.

Many large animals are closer anatomically and physiologically to humans, can be outbred, show many clinical similarities to the human infection, and very importantly have ACE2 receptors recognized by the SARS-CoV-2 virus. There are several drawbacks, however. No animal model has manifested clinical symptoms as severe as observed in humans, especially regarding the mortality rate. Only a few studies could be found which involved an aged animal, ${ }^{34,40}$ where even then no fatality was observed. We conjecture that environmental factors could be at play as animals are typically kept in well-controlled indoor environments, whereas patient morbidity correlates with regions of increased air pollution. ${ }^{57}$ The results of therapeutic studies on animals housed in sterile conditions should therefore be interpreted cautiously. 
Also, the animal models discussed above have been tested with SARS-CoV-2 strains which were clinically available from local human subjects. Recent reports show as many as fourteen mutated variants of the spike proteins from different geographical locations, some of which are more virulent than the others. ${ }^{32}$ Studies on one or two specific local strains may be hard to extrapolate to a global scale. As with many large animal studies, most done for SARS-CoV-2 were limited in the number of animals tested compared to gold-standard preclinical rodent studies, except a large study of DNA vaccine candidates on 35 adult rhesus macaques. ${ }^{61}$ These limited numbers, coupled with a lack of investigation into the duration of protection afforded by neutralizing antibodies, leave much work in the development and testing of anti-viral treatments and vaccination strategies.

Drawbacks notwithstanding, a wide range of animal models with ACE2 receptors and serine proteases susceptible to SARS-CoV-2 are available to study emerging technologies in the global fight against COVID-19. With the rapid pace of research on this topic, we can expect the development of new animal models with their own advantages and shortcomings and generation of new and better knowledge about the novel disease to arrive every day.

\section{ACKNOWLEDGMENTS}

This work was supported by the National Institute of General Medical Sciences (NIH) through Grant R35GM128831 to CSS.

\section{CONFLICT OF INTEREST}

Nothing to disclose.

\section{REFERENCES}

\footnotetext{
${ }^{1}$ Banerjee, A., et al. Generation and characterization of Eptesicus fuscus (Big brown bat) kidney cell lines immortalized using the Myotis polyomavirus large T-antigen. $J$. Virol. Methods 237:166-173, 2016.

${ }^{2}$ Bao, L., et al. The pathogenicity of SARS-CoV-2 in hACE2 transgenic mice. Nature 2020. https://doi.org/10. 1038/s41586-020-2312-y.

${ }^{3}$ Bao, L., et al. Lack of reinfection in rhesus macaques infected with SARS-CoV-2. Microbiology 2020. https://doi. org/10.1101/2020.03.13.990226.

${ }^{4}$ Baxter, V. K., and D. Griffin. Animal models: No model is perfect, but many are useful. In: Viral Pathogenesis From Basics to Systems Biology, edited by N. Nathanson, M. Katze, M. Korth, and G. L. Law. London: Elsevier, 2016 , pp. $125-138$.
}

${ }^{5}$ Bility, M. T., et al. Hepatitis B virus infection and immunopathogenesis in a humanized mouse model: induction of human-specific liver fibrosis and M2-like macrophages. PLoS Pathog 10:e1004032, 2014.

${ }^{6}$ Blanco-Melo, D., et al. Imbalanced host response to SARS-CoV-2 drives development of COVID-19. Cell 181:1036-1045.e9, 2020.

${ }^{7}$ Brehm, M. A., M. V. Wiles, D. L. Greiner, and L. D. Shultz. Generation of improved humanized mouse models for human infectious diseases. J Immunol Methods 410:317, 2014.

${ }^{8}$ Cavanagh, D. Coronaviruses in poultry and other birds. Avian Pathol 34:439-448, 2005.

${ }^{9}$ Chan, J. F.-W., et al. Treatment with lopinavir/ritonavir or interferon- $\beta 1 \mathrm{~b}$ improves outcome of MERS-CoV infection in a nonhuman primate model of common marmoset. $J$. Infect. Dis. 212:1904-1913, 2015.

${ }^{10}$ Chan, J. F.-W., et al. Simulation of the clinical and pathological manifestations of Coronavirus Disease 2019 (COVID-19) in golden Syrian hamster model: implications for disease pathogenesis and transmissibility. Infect. Dis. Clin 2020. https://doi.org/10.1093/cid/ciaa325.

${ }^{11}$ Chandrashekar, A., et al. SARS-CoV-2 infection protects against rechallenge in rhesus macaques. Science 2020. htt ps://doi.org/10.1126/science.abc4776.

${ }^{12}$ Curran, M., et al. Recent advancements and applications of human immune system mice in preclinical immuno-oncology. Toxicol Pathol 48:302-316, 2020.

${ }^{13}$ Decaro, N., and A. Lorusso. Novel human coronavirus (SARS-CoV-2): A lesson from animal coronaviruses. Vet. Microbiol. 244:108693, 2020.

${ }^{14}$ Deng, W., et al. Ocular conjunctival inoculation of SARSCoV-2 can cause mild COVID-19 in Rhesus macaques. BioRxiv 2020. https://doi.org/10.1101/2020.03.13.990036.

${ }^{15}$ Dyal, J. W. COVID-19 among workers in meat and poultry processing facilities-19 states. MMWR Morb Mortal Wkly Rep 69(27):887-892, 2020.

${ }^{16}$ Elahi, S., J. Holmstrom, and V. Gerdts. The benefits of using diverse animal models for studying pertussis. Trends in Microbiol. 15:462-468, 2007.

${ }^{17}$ Fouchier, R. A. M., et al. Koch's postulates fulfilled for SARS virus. Nature 423:240-240, 2003.

${ }^{18}$ Golding, H., S. Khurana, and M. Zaitseva. What is the predictive value of animal models for vaccine efficacy in humans? The importance of bridging studies and speciesindependent correlates of protection. Cold Spring Harb. Perspect. Biol. 10:a028902, 2018.

${ }^{19}$ González, J. M., P. Gomez-Puertas, D. Cavanagh, A. E. Gorbalenya, and L. Enjuanes. A comparative sequence analysis to revise the current taxonomy of the family Coronaviridae. Arch. Virol. 148:2207-2235, 2003.

${ }^{20}$ Guan, W.-J., et al. Clinical characteristics of coronavirus disease 2019 in China. N. Engl. J. Med. 382:1708-1720, 2020.

${ }^{21}$ Gustafson, K. D., M. G. Hawkins, T. L. Drazenovich, R. Church, S. A. Brown, and H. B. Ernest. Founder events, isolation, and inbreeding: Intercontinental genetic structure of the domestic ferret. Evol. Appl. 11:694-704, 2018.

${ }^{22}$ Halfmann, P. J., et al. Transmission of SARS-CoV-2 in domestic cats. N. Engl. J. Med. 2020. https://doi.org/10. 1056/NEJMc2013400.

${ }^{23}$ Hamilton, I. M., and R. M. R. Barclay. Patterns of daily torpor and day-roost selection by male and female big brown bats (Eptesicus fuscus). Can. J. Zool. 72:744-749, 1994. 
${ }^{24}$ Hoffmann, M., et al. SARS-CoV-2 cell entry depends on ACE2 and TMPRSS2 and is blocked by a clinically proven protease inhibitor. Cell 181:271-280.e8, 2020.

${ }^{25}$ Hosie, M. J., et al. SARS coronavirus 2 and cats. Eur: Eur. Advis. Board Cat Dis, 2020. https://doi.org/10.2807/15607917.ES.2020.25.23.2001005.

${ }^{26}$ IDEXX SARS-CoV-2 (COVID-19) RealPCR Test. [Accessed 2020 Jun 3]. Available from: https://www.idexx.co $\mathrm{m} / \mathrm{en} /$ veterinary/reference-laboratories/idexx-sars-cov-2-co vid-19-realpcr-test/.

${ }^{27}$ Imai, Y., et al. Angiotensin-converting enzyme 2 protects from severe acute lung failure. Nature 436:112-116, 2005.

${ }^{28}$ Iwata-Yoshikawa, N., T. Okamura, Y. Shimizu, H. Hasegawa, M. Takeda, and N. Nagata. TMPRSS2 contributes to virus spread and immunopathology in the airways of murine models after coronavirus infection. J. Virol. 2019. h ttps://doi.org/10.1128/JVI.01815-18.

${ }^{29} \mathrm{Kim}, \mathrm{K}$. C., et al. A simple mouse model for the study of human immunodeficiency virus. AIDS Res. Hum. Retroviruses. 32:194-202, 2016.

${ }^{30} \mathrm{Kim}$, Y.-I., et al. Infection and rapid transmission of SARS-CoV-2 in ferrets. Cell Host Microbe 27:704-709.e2, 2020.

${ }^{31}$ Kiros, T. G., B. Levast, G. Auray, S. Strom, J. van Kessel, and V. Gerdts. The importance of animal models in the development of vaccines. Innov. Vaccinol. 29:251-264, 2012.

${ }^{32}$ Korber, B., et al. Spike mutation pipeline reveals the emergence of a more transmissible form of SARS-CoV-2. BioRxiv 2020. https://doi.org/10.1101/2020.04.29.069054.

${ }^{33}$ Liu, K., Y. Chen, R. Lin, and K. Han. Clinical features of COVID-19 in elderly patients: A comparison with young and middle-aged patients. J. Infect. 80:e14-e18, 2020.

${ }^{34} \mathrm{Lu}$, S., et al. Comparison of SARS-CoV-2 infections among 3 species of non-human primates. BioRxiv 2020. h ttps://doi.org/10.1101/2020.04.08.031807.

${ }^{35} \mathrm{McCray}, \mathrm{P}$. B., et al. Lethal infection of K18-hACE2 mice infected with severe acute respiratory syndrome coronavirus. J. Virol. 81:813-821, 2007.

${ }^{36}$ Mota, J., and R. Rico-Hesse. Humanized mice show clinical signs of dengue fever according to infecting virus genotype. J. Virol. Am. Soc. Microbiol. J. 83:8638-8645, 2009.

${ }^{37}$ Munster, V. J., et al. Respiratory disease and virus shedding in rhesus macaques inoculated with SARS-CoV-2. BioRxiv 2020. https://doi.org/10.1101/2020.03.21.001628.

${ }^{38}$ Pearson, T., D. L. Greiner, and L. D. Shultz. Creation of "humanized" mice to study human immunity. Curr: Protoc. Immunol, 2008. https://doi.org/10.1002/0471142735.i $\mathrm{m} 1521 \mathrm{~s} 81$.

${ }^{39}$ Roberts, A., et al. Severe acute respiratory syndrome coronavirus infection of golden Syrian hamsters. J. Virol. 79:503-511, 2005.

${ }^{40}$ Rockx, B., et al. Comparative pathogenesis of COVID-19, MERS, and SARS in a nonhuman primate model. Science 368:1012-1015, 2020.

${ }^{41}$ Shan, C., Y.F. Yao, X.L. Yang, Y.W. Zhou, J. Wu, and G. Gao. Infection with Novel Coronavirus (SARS-CoV-2) Causes Pneumonia in the Rhesus Macaques [Internet]. Preprint, 2020. Available from: https://www.researchsqua re.com/article/rs-15756/v1.

${ }^{42}$ Shi, J., et al. Susceptibility of ferrets, cats, dogs, and other domesticated animals to SARS-coronavirus 2. Science 368:1016-1020, 2020.
${ }^{43}$ Shoenfeld, Y. Corona (COVID-19) time musings: Our involvement in COVID-19 pathogenesis, diagnosis, treatment and vaccine planning. Autoimmun. Rev. 19:102538, 2020.

${ }^{44}$ Shultz, L. D., M. A. Brehm, J. V. Garcia-Martinez, and D. L. Greiner. Humanized mice for immune system investigation: Progress, promise and challenges. Nat. Rev. Immunol. 12:786-798, 2012.

${ }^{45} \mathrm{Sia}$, S.F. et al. Pathogenesis and transmission of SARSCoV-2 in golden hamsters. Nature 1-7, 2020.

${ }^{46}$ Sikes, R. S., and W. L. Gannon. Guidelines of the American Society of Mammalogists for the use of wild mammals in research. J. Mammal. 92:235-253, 2011.

${ }^{47}$ Sit, T.H.C. et al. Infection of dogs with SARS-CoV-2. Nature Springer US, 1-12, 2020.

${ }^{48}$ Smits, S. L., et al. Exacerbated innate host response to SARS-CoV in aged non-human primates. PLoS Pathog. 6:e1000756-e1000756, 2010.

${ }^{49}$ Soldatov, V. O., M. V. Kubekina, Y. Y. Silaeva, A. V. Bruter, and A. V. Deykin. On the way from SARS-CoVsensitive mice to murine COVID-19 model. Res. Results Pharmacol. 6(2):1-7, 2020.

${ }^{50}$ Subbarao, K., and A. Roberts. Is there an ideal animal model for SARS? Trends Microbiol. 14:299-303, 2006.

${ }^{51}$ Tseng, C.-T. K., et al. Severe acute respiratory syndrome coronavirus infection of mice transgenic for the human angiotensin-converting enzyme 2 virus receptor. J. Virol. 81:1162-1173, 2007.

${ }^{52}$ van den Brand, J. M. A., et al. Pathology of experimental SARS coronavirus infection in cats and ferrets. Vet. Pathol. 45:551-562, 2008.

${ }^{53}$ Wahl, A., et al. Precision mouse models with expanded tropism for human pathogens. Nat. Biotechnol. 37:11631173,2019

${ }^{54}$ Wan, Y., J. Shang, R. Graham, R. S. Baric, and F. Li. Receptor recognition by the novel coronavirus from $\mathrm{Wu}-$ han: An analysis based on decade-long structural studies of SARS Coronavirus. J. Virol. 94(7):e0012720, 2020.

${ }^{55}$ Wang, Q. hACE2 transgenic mouse model for coronavirus (COVID-19) research. The Jackson Laboratory. 2020 [cited 2020 Jun 2]. Available from: https:/www.jax.org/news-a nd-insights/2020/february/introducing-mouse-model-for-c orona-virus.

${ }^{56}$ Weingartl, H., et al. Immunization with modified vaccinia virus Ankara-based recombinant vaccine against severe acute respiratory syndrome is associated with enhanced hepatitis in ferrets. J. Virol. 78:12672-12676, 2004.

${ }^{57}$ Wu, X., R. C. Nethery, B. M. Sabath, D. Braun, and F. Dominici. Exposure to air pollution and COVID-19 mortality in the United States: A nationwide cross-sectional study. medRxiv 2020. https://doi.org/10.1101/2020.04.05.2 0054502 .

${ }^{58}$ Yang, X.-H., et al. Mice transgenic for human angiotensinconverting enzyme 2 provide a model for SARS coronavirus infection. Comp. Med. 57:450-459, 2007.

${ }^{59}$ Ye, Q., B. Wang, and J. Mao. The pathogenesis and treatment of the 'Cytokine Storm' in COVID-19. J. Infect. 80:607-613, 2020.

${ }^{60}$ Yong, K. S. M., et al. Bat-mouse bone marrow chimera: a novel animal model for dissecting the uniqueness of the bat immune system. Sci. Rep. 8:4726, 2018.

${ }^{61} \mathrm{Yu}, \mathrm{J}$., et al. DNA vaccine protection against SARS-CoV-2 in rhesus macaques. Science 2020. https://doi.org/10.1126/ science.abc6284. 
${ }^{62} \mathrm{Yu}$, P., et al. Age-related rhesus macaque models of COVID-19. Anim. Models Exp. Med. 3:93-97, 2020.

${ }^{63}$ Zhang, Q. et al. SARS-CoV-2 neutralizing serum antibodies in cats: a serological investigation. Microbiology, 2020.

${ }^{64}$ Zhou, P., et al. Unlocking bat immunology: establishment of Pteropus alecto bone marrow-derived dendritic cells and macrophages. Sci. Rep. 6:38597, 2016.
Publisher's Note Springer Nature remains neutral with regard to jurisdictional claims in published maps and institutional affiliations. 